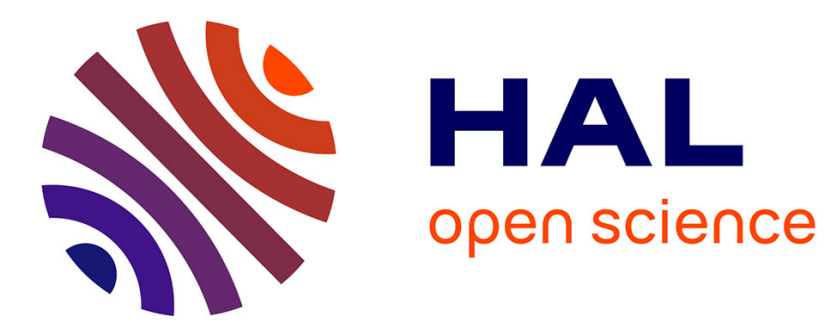

\title{
Les entreprises sociales à la recherche de leur légitimité Constance Perrin-Joly
}

\section{To cite this version:}

Constance Perrin-Joly. Les entreprises sociales à la recherche de leur légitimité. Revue internationale des études du développement, 2021, Nº 245 (1), pp.115-144. 10.3917/ried.245.0115 . halshs-03213055

\section{HAL Id: halshs-03213055 \\ https://shs.hal.science/halshs-03213055}

Submitted on 3 May 2021

HAL is a multi-disciplinary open access archive for the deposit and dissemination of scientific research documents, whether they are published or not. The documents may come from teaching and research institutions in France or abroad, or from public or private research centers.
L'archive ouverte pluridisciplinaire HAL, est destinée au dépôt et à la diffusion de documents scientifiques de niveau recherche, publiés ou non, émanant des établissements d'enseignement et de recherche français ou étrangers, des laboratoires publics ou privés. 
REVUE INTERNATIONALE

DES ÉTUDES DU DÉVELOPPEMENT

\section{- L'entrepreneuriat en Afrique}

Sous la direction de Quentin Chapus, Jean-Philippe Berrou

et Yvette Onibon Doubogan

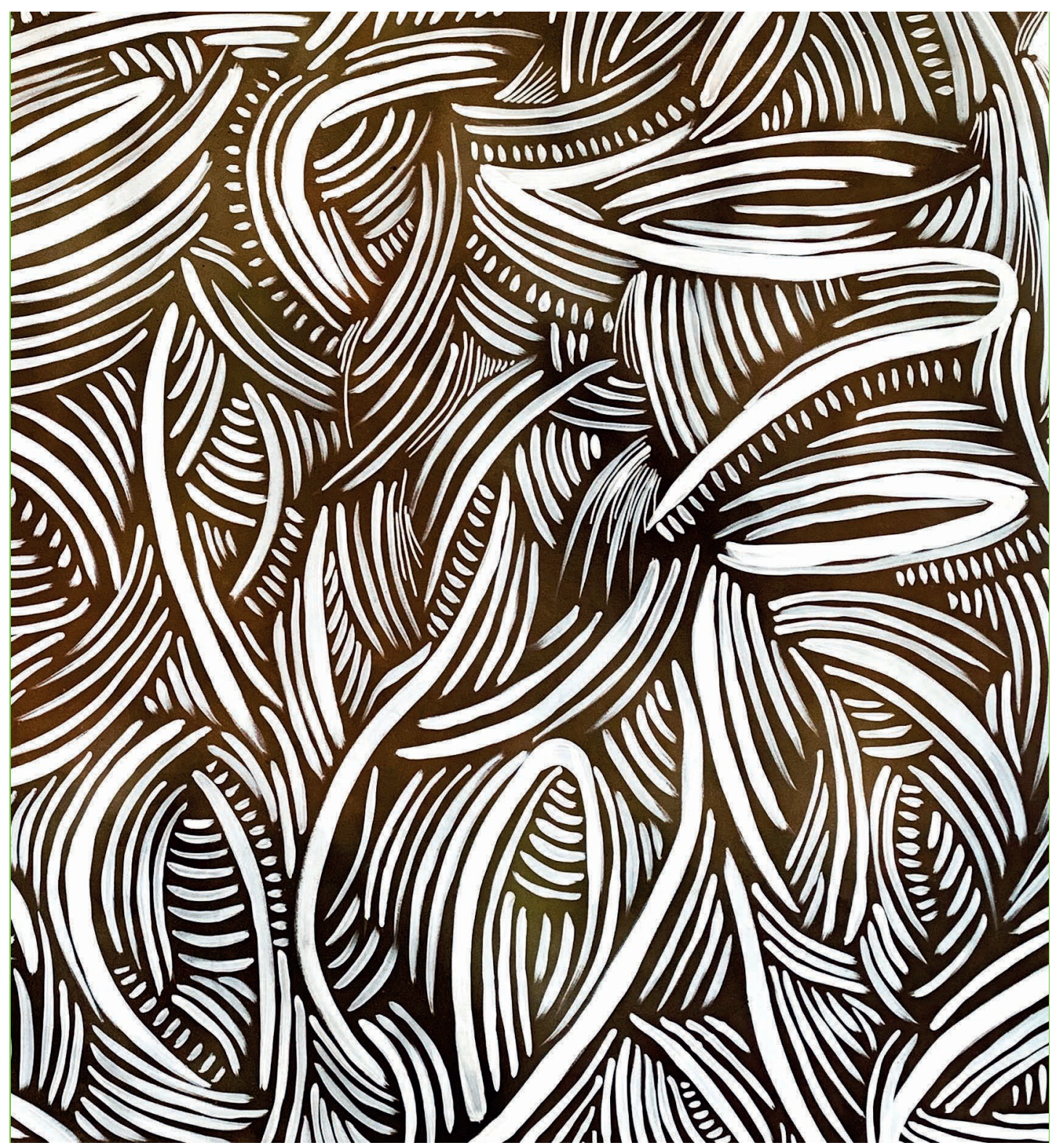




\title{
Les entreprises sociales à la recherche de leur légitimité
}

\author{
Frictions autour du projet de développement \\ éthiopien et tentatives d'extraversion
}

\begin{abstract}
Constance Perrin-Joly
RÉSUMÉ

L'engagement des entreprises sociales dans le développement de l'Éthiopie s'inscrit dans une forme néolibérale de gouvernement, reposant sur une responsabilisation d'acteurs individuels sommés de se mêler du bien commun, démontrant en creux l'incapacité de l'État à le faire. Ce positionnement amène les entreprises sociales à entrer en tension avec l'État développemental éthiopien, détenteur du monopole du développement. L'entrepreneur social est perçu comme se situant en marge des politiques publiques d'industrialisation, d'une part, et porte un cadre idéologique échappant au contrôle au gouvernemental, d'autre part. L'Éthiopie constitue ainsi un point d'observation privilégié des stratégies de légitimation d'entreprises sociales en quête de reconnaissance institutionnelle, mobilisant les ressources de l'extraversion tout en valorisant leur ancrage local.
\end{abstract}

\section{MOTS-CLÉS}

entreprise sociale, ethnographie, entrepreneur, sociologie, Éthiopie 



\section{Introduction}

Tentreprise est de plus en plus considérée par de nombreux gouvernements nationaux et par les instances supranationales (Organisation de coopération et de développement économiques - OCDE -, Banque mondiale...) comme un acteur central du développement des Suds. Toutefois, ses conditions d'exercice ont fait l'objet d'une contestation croissante de la part d'organisations non gouvernementales (ONG), qui dénoncent les affaires de corruption ou le scandale des sweatshops (conditions de travail indécentes dans des usines du Honduras sous-traitantes pour grandes marques de textile américaines au milieu des années 1990 (Barraud de Lagerie, 2012), effondrement d'une usine au Bangladesh en 2005 (Barraud de Lagerie, 2011). L’idée que la main invisible du marché assurerait automatiquement le bienêtre des populations a été largement délégitimée et les entreprises ont été de plus en plus enjointes à ne pas se limiter à créer de l'activité économique au Sud, mais à répondre à des obligations morales, par la mise en place de politiques dites « citoyennes » (développement durable, entrepreneuriat inclusif, responsabilité sociale des entreprises).

Dans ce contexte, la promotion des entreprises dites « sociales » a pu être présentée comme une voie pour un développement économique durable au Nord comme au Sud. C'est le cas en Éthiopie, qui a accueilli le Social Enterprise World Forum (SEWF) à Addis-Abeba, en 2019.

Issues de différentes traditions nationales (Defourny, 2011 ; Defourny \& Nyssens, 2010 ; Kerlin, 2006 ; Le Velly, 2014), les contours de la catégorie « entreprise sociale » varient. On distingue généralement la tradition angloaméricaine axée sur les ONG développant des activités à but lucratif, de la tradition d'Europe continentale qui regroupe des structures à buts lucratif et non lucratif (comme les coopératives) mais qui insiste sur leur organisation démocratique interne (Young \& Salamon, 2002 ; Defourny \& Nyssens, 2010 ; Kerlin, 2006). Une deuxième école de pensée américaine coexiste (American Social Innovation School), faisant de l'entrepreneur social avant tout un artisan du changement (Dees \& Battle Anderson, 2006 ; Bacq \& Janssen, 2011). Elle est particulièrement prégnante en Éthiopie, où, on le verra, différents entrepreneurs ont été distingués par la fondation Schwab qui en est une des promotrices. La définition de l'entreprise sociale doit en effet également être saisie en fonction des intérêts des acteurs qui promeuvent ce dispositif 
(Dart, 2004 ; Nicholls, 2010 ; Huybrechts et al., 2012 ; Bacq \& Janssen, 2011). Or, en Éthiopie, le British Council a joué un rôle de premier plan ces dernières années pour promouvoir la catégorie « entreprise sociale », diffusant une approche britannique en complément de la tradition américaine.

Si nous avons pu rencontrer des ONG développant des activités marchandes, l'enquête sur laquelle se fonde cet article (voir infra) nous a davantage amenés à interroger des entrepreneurs dont les activités sont situées dans un «spectre hybride » entre les entreprises sociales et les entreprises privées classiques (Alter, 2002) et pour qui ces différentes institutions étrangères sont sollicitées comme des instances de légitimation. Si certaines entreprises labellisées sociales font du profit et rémunèrent d'éventuels actionnaires, la rentabilité économique est cependant pensée comme une condition nécessaire mais non suffisante. Sa dimension sociale est mise au centre du projet entrepreneurial, dimension censée être la garantie de leur participation au bien commun.

En appeler à la responsabilité « sociale » des entreprises revient à prendre acte d'une forme néolibérale de gouvernement des populations, reposant sur une responsabilisation d'acteurs individuels (Clarke, 2004) non seulement sommés de réparer leurs externalités négatives mais aussi de se mêler du bien commun. Confier aux entreprises la définition et les moyens d'assurer ce «bien commun » fait courir le risque de privatiser « les pratiques de développement », démontrant en creux l'incapacité de l'État à le faire (Connan, 2014: 136). C'est d'autant plus vrai pour les entreprises accompagnées par le British Council, qui présente depuis 2005 les entreprises sociales « comme réponse à la défaillance de l'État ${ }^{1}$ » (Teasdale, 2011: 112, traduction de l'auteure). La forte implication d'institutions internationales, tout comme les nécessaires stratégies d'extraversion sur lesquelles repose le modèle économique de nombre d'entreprises sociales en Éthiopie, viennent par ailleurs renforcer leur dépendance vis-à-vis des pays du centre (c'est-àdire les pays du Nord).

Or, en Éthiopie, le développement économique constitue à la fois le monopole du gouvernement, qui mise sur un État développemental (Johnson,

1 "As a response to state failure ". 
1982 ; Clapham, 2018), et une ressource essentielle pour le maintien du parti au pouvoir et la mobilisation des masses (Mains, 2012). Ce dernier définit strictement la participation du secteur privé, encourageant essentiellement les industries exportatrices et/ou consommatrices de main-d'œuvre (Lefort, 2015 ; Altenburg, 2O1O ; Oqubay Arkebe, 2015). Autoritaire (Morelle \& Planel, 2018 ; Bach, 2016), en dépit d'un relatif desserrement avec l'arrivée d'un nouveau premier ministre (Aisserge \& Bach, 2018), le gouvernement éthiopien semble peu favorable à un entrepreneur social qui se situe en marge de ses politiques d'industrialisation et risque de porter un cadre idéologique échappant à son contrôle. L'Éthiopie constitue ainsi un point d'observation privilégiée à la fois des stratégies de légitimation d'entreprises sociales encore en quête de reconnaissance institutionnelle (Nicholls, 2010 ; Dart, 2004), tout comme des tensions qui traversent les politiques éthiopiennes de développement.

Nous détaillerons en premier lieu la façon dont l'État développemental éthiopien encadre le rôle dévolu au secteur privé dans ses politiques économiques. Parce qu'elles valorisent l'entrepreneuriat pour lui-même et non comme « moyen », les entreprises sociales se mettent en porte-à-faux avec le projet de développement du gouvernement (partie 2). Il s'agit alors pour elles de puiser dans différentes stratégies de l'extraversion pour gagner en légitimité (partie 3).

Les données mobilisées dans cet article s'inscrivent dans le cadre d'une enquête ethnographique sur l'engagement moral des entrepreneurs en Éthiopie. Commencée en 2015, cette étude croise une quarantaine d'entretiens biographiques avec des entrepreneuses et entrepreneurs étrangers ou éthiopiens ${ }^{2}$ mettant en ouvre des politiques sociales diverses, avec des observations au sein des associations d'entreprises, et des entretiens avec les salariés dans des entreprises sélectionnées. Dans ce cadre, ont été rencontrés une douzaine d'entrepreneurs sociaux, dont l'objectif social de l'entreprise est présenté par eux-mêmes comme central dans le projet organisationnel

2 Nous soulignons ici la diversité de genre de notre panel, variable importante dans la construction de l'enquête mais peu mobilisée dans le présent papier. C'est pour cette raison et par souci de simplification que nous ne la mobilisons pas dans le reste de l'article. Les entretiens ont eu lieu en français ou en anglais, sauf pour la majorité des salariés avec lesquels nous nous sommes entretenus en amharique, la plupart du temps avec un interprète qui nous a accompagné tout au long de l'enquête. 
(par opposition à des entreprises qui, à côté de leur activité économique, conduiraient des activités de responsabilité sociale ou de philanthropie). Ces entretiens ont été complétés par la visite des entreprises considérées et, dans deux cas, la rencontre des salariés lors d'entretiens semi-directifs.

Cette enquête qualitative ne se fonde pas sur un échantillon statistiquement représentatif du tissu mal identifié des entreprises sociales en Éthiopie. Une étude du British Council a estimé à 55 ooo le nombre d'entreprises sociales opérant dans le pays, en se basant sur la définition suivante :

Les entreprises sociales sont des entreprises qui s'attaquent aux problèmes sociaux et environnementaux. Elles créent des emplois et génèrent des revenus comme les autres entreprises, mais au lieu de canaliser leurs bénéfices vers leurs propriétaires, elles les réinvestissent pour soutenir leur mission sociale. Ce faisant, elles améliorent la vie des gens dans nos communautés et nos sociétés $^{3}$. (British Council, 2016, traduction de l'auteure)

Il est fort probable que le dénombrement des entreprises sociales est largement surestimé, cette institution ayant intérêt à agglomérer sous ce label qu'elle promeut une diversité d'organisations. Il s'agirait en large majorité de petites entreprises (Micro and Small entreprises - MSE), employant en moyenne 21 salariés, représentant ainsi $10 \%$ de ce tissu organisationnel (les MSE sont évaluées par l'agence publique qui les chapeaute à un peu moins de 500 ooo au mitan des années 2010). Les entreprises sociales en Éthiopie constituent ainsi une minorité dans un secteur privé par ailleurs limité, mais l'un et l'autre sont en constante augmentation depuis 2010. Notre démarche se fonde sur un panel contrasté de situations pour construire une représentativité sociologique (Hamel, 20oo) de l'entreprise sociale dans le contexte économique éthiopien. Les cas sont contrastés au regard de leur réussite économique (certaines entreprises enquêtées peinent à lancer leur production, quand d'autres ont pignon sur rue et emploient jusqu'à plus de 3 oo salariés). Ils poursuivent deux types d'objets, se focalisant soit sur la question de l'emploi local (y compris de victimes de discrimination comme

3 "Social enterprises are businesses that tackle social and environmental problems. They create jobs and generate income like other businesses, but instead of channeling their profits to owners they reinvest them to support their social mission. In doing so, they are improving people's lives in our communities and societies. " 
les personnes en situation de handicap), soit sur des services jugés « utiles » à la population locale (par exemple en proposant des produits alimentaires ayant de meilleures qualités nutritionnelles pour lutter contre la malnutrition) ou/et valorisant les atouts du pays, et ce dans des secteurs variés (l'artisanat, en particulier dans le secteur textile, la maroquinerie, ou encore le tourisme). Enfin, même si elles sont toutes de droit éthiopien, les entreprises étudiées permettent de comparer des entrepreneurs éthiopiens et étrangers.

Une première vague d'entretiens a eu lieu entre 2016 et 2018, les enquêtés ayant été identifiés via des associations d'entreprises étrangères (non spécialisées dans l'entrepreneuriat social) ou par le bouche-à-oreille et la méthode de la boule de neige (un contact en fournissant un autre). La tenue du SEWF a rendu plus visible la tentative d'institutionnalisation de ce « secteur » émergent. Une seconde vague d'entretiens avec des entrepreneurs tous liés au British Council ou à l'association des entreprises sociales éthiopiennes a eu lieu entre 2018 et 2019. Elle a permis d'observer ce processus en cours. Érigés en hérauts de l'entreprise sociale éthiopienne, ces entrepreneurs sont nombreux à avoir donné des conférences ou participé à des entretiens que nous mobilisons également pour analyser leur discours de légitimation.

Ces différentes phases d'enquête nous ont permis de regrouper des entrepreneurs qui revendiquent tous une finalité sociale pour leur entreprise. Cependant, certains ne s'appuient sur aucun dispositif institutionnel spécifique (c'est le cas de nos enquêtés de la "première vague »), alors que d'autres (rencontrés dans un second temps) s'inscrivent dans des dispositifs dont ils mobilisent les ressources discursives et institutionnelles pour faire reconnaître leur participation au développement.

\section{Des entrepreneurs mis au service de l'État développemental éthiopien}

L'autoritarisme du régime et l'engagement dans une politique développementale font du gouvernement un acteur central dans l'encadrement de l'économie, notamment dans la distinction entre figures acceptables (development-oriented investor) et catégories repoussoirs de l'entrepreneuriat (rent-seeker). L'autoritarisme du régime repose sur la monopolisation du 
pouvoir par le même parti (le Front démocratique révolutionnaire du peuple éthiopien, en anglais : Ethiopian People's Revolutionary Democratic Front - EPDRF) depuis la chute du Derg (junte militaire au pouvoir à partir de la destitution de l'Empereur en 1974) en 1991. D'abord engagé dans une politique économique se revendiquant du maoïsme et du marxisme albanien (Young, 1997 ; Clapham, 2006 ; Lefort, 2015), la remise en cause du Parti par la population lors des élections de 2005 a accéléré son orientation vers une économie dirigée (Aalen \& Tronvoll, 2009; Lefort, 2012). Le Premier ministre éthiopien Meles Zenawi (1991-2012), artisan de cette politique (De Waal, 2013 ; Zenawi Meles, 2012), a alors initié un mode de gouvernement économique dirigé par l'État et inspiré des modèles asiatiques, l'État développemental (Johnson, 1982). Il a ainsi préféré le consensus de Beijing (Ramo, 2004) au consensus de Washington qui impose un processus de démocratisation politique, alors peu compatible avec l'autoritarisme du régime (Bach, 2016). Si, depuis les années 2010, le secteur privé jusqu'alors confidentiel n'a cessé de se développer, l'entreprise constitue une figure ambiguë, «le secteur privé n'est encouragé que dans la mesure où il correspond aux priorités définies par l'État-parti » (Bach, 2011: 469).

\subsection{Des investisseurs étrangers soumis au cadre de l'État développemental éthiopien}

Faute de ressources minières ou pétrolifères, mobiliser le capital nécessaire à une ambitieuse politique publique de développement et de construction d’infrastructures dans un pays pauvre aux faibles ressources fiscales nécessitait de trouver d'autres sources de rentes étatiques. Ces dernières ont pu être directes (via des entreprises publiques monopolistiques) ou indirectes via des fonds d'investissement propriété du parti au pouvoir (Vaughan \& Mesfin Gebremichael, 2011). Le financement de la politique de développement s'est également appuyé sur une logique d'extraversion à l'instar d'autres pays africains (Bayart, 1989), captant la manne des aides internationales, les institutions étrangères soutenant volontiers un pays considéré comme une des rares nations stables de la région (Dereje Feyissa, 2012 ; Fantini \& Puddu, 2016).

Le régime a enfin tenté d'attirer les investisseurs étrangers, à la fois pourvoyeurs d'emplois pour le deuxième pays le plus peuplé d'Afrique et de devises étrangères grâce aux exportations (Clapham, 2018). Ceux-ci ont trouvé en Éthiopie un réservoir de main-d'œuvre à bas coût mais aussi 
une politique d'incitation fiscale, une relative stabilité politique et des infrastructures ambitieuses. Les parcs industriels construits par l'État et qui ont ouvert leurs portes dès 2017 ont été conçus comme un des fleurons de la politique industrielle éthiopienne (Aspen, 2020 ; Barrett \& Baumann-Pauly, 2019). Ces parcs accueillent dans des hangars identiques des entreprises manufacturières exportatrices notamment dans le domaine du textile et de la maroquinerie, elles bénéficient d'un guichet unique pour leurs démarches administratives, de facilités pour l'exportation et d'un accès prioritaire aux devises. L'ensemble de cette politique en faveur de l'industrialisation vise donc des investisseurs importants, amenés à employer plusieurs milliers de salariés éthiopiens dans des secteurs choisis. La libéralisation est en effet sélective, l'État continue à contrôler les secteurs jugés stratégiques (les banques, les assurances, les télécommunications et la fourniture d'électricité sont toujours sous la coupe étatique), sa monnaie (par un contrôle du taux de change et une maîtrise drastique de sa convertibilité) et l'octroi de terres, dont l'État conserve la propriété.

Jusqu'au mitan des années 2010, attirer des entreprises étrangères n'est pas présenté comme un choix mais comme "un mal nécessaire » (Chaponnière, 2018), « l'Éthiopie n'avait pas d'autre choix "pour survivre” que de procéder à un "changement structurel” afin "d'utiliser l'économie de marché4” » (EPRDF, 2006, cité par Lefort, 2012: 682, traduction de l'auteure).

Le secteur public reste très majoritaire et tire la croissance grâce aux investissements en infrastructures et à des entreprises pilotes comme Ethiopian Airlines (Altenburg, 2010). Le secteur privé est par ailleurs dominé par des entreprises liées au Parti - via des fonds d'investissement régionaux comme EFFORT pour le Tigray, région au Nord du pays et ethnie longtemps dominante au sein de la coalition de l'EPRDF (Vaughan \& Mesfin Gebremichael, 2011) - et par le conglomérat MIDROC. Ce dernier est détenu par l'homme d'affaires éthio-saoudien Mohammed Al Amudi, premier employeur privé du pays, connu pour ses liens étroits avec le parti au pouvoir (Calvary, 2014). Dans ce contexte, les autres investisseurs du secteur privé, locaux ou étrangers, pèsent peu dans l'arène politique (Vaughan \& Tronvoll, 2013: 77).

4 "Ethiopia had no other choice 'to survive' than to carry out a 'structural change' in order 'to employ free market economy'. " 
La mort de l'instigateur du tournant développemental de la politique économique éthiopienne, Meles Zenawi, en 2012, puis l'avènement au pouvoir d'un nouveau Premier ministre, Abiy Ahmed en 2018, ont pu amener certains observateurs à identifier une conversion du nouveau gouvernement au néolibéralisme (Planel \& Labzaé, 2018). Le caractère ambivalent du gouvernement éthiopien n'est cependant pas nouveau, nombre de chercheurs ont souligné sa capacité à mobiliser des financements des agences internationales en promouvant une politique néolibérale (Mains, 2007) et à tenir ensemble ouverture au marché et dirigisme économique. Abiy Ahmed, socialisé dans les rangs de l'EPRDF (Aisserge \& Bach, 2018 ; Aalen, 202O), a certes affirmé « être capitaliste » (Nallet, 2019) et reconnu avoir «besoin du secteur privé ». Pourtant, les entrepreneurs enquêtés soulignent le peu de changement dans le climat des affaires, les demandes récurrentes de «sponsors » (comme pour le projet de verdissement de la ville d'Addis-Abeba lancé par Abiy Ahmed), la multiplication des contrôles fiscaux tatillons aux résultats contestables mais pour lesquels les recours sont complexes, longs et périlleux.

Au-delà d'une relative libéralisation de la parole et de la vie politique, la fin des années 2010 ne permet pas d'affirmer que la donne a fondamentalement changé. Face à un endettement public croissant (61\% du PIB en 2018), les entreprises étrangères semblent être considérées comme une ressource parmi d'autres, sollicitées tant qu'elles s'inscrivent dans le cadre développemental imposé par l'État, que ce soit en investissant, en apportant des devises étrangères ou en finançant directement des politiques publiques.

\subsection{L'entrepreneuriat comme moyen d'enrôlement dans le développement}

Le bon entrepreneur, défini comme « development-oriented » est, aux yeux du gouvernement, un investisseur qui s'inscrit dans les projets du Parti. L'entrepreneuriat n'est pas une fin en soi, c'est un moyen pour atteindre des objectifs économiques (devenir un pays à revenu médian) mais aussi une façon d'encadrer la population. Les dispositifs publics en faveur d'un entrepreneuriat « de nécessité » sont ainsi présentés comme un moyen de sortir la jeune population urbaine de la pauvreté. Les entrepreneurs considérés sont en priorité des indépendants ou des micro-entreprises, au capital inférieur à quelques milliers de birrs, des petites activités urbaines ou complémentaires au travail 
agricole enjointes à sortir de l'informalité et dont le revenu ne permet souvent que la subsistance de leur propriétaire. Ces actions sont largement soutenues par des organisations internationales comme l'Organisation internationale du travail (OIT) depuis le début des années 2000 (Zewde \& Associates, 2002 ; Stevenson \& St-Onge, 2005). Le National Development Programme to Accelerate Sustainable Development to Eradicate Poverty (PASDEP) en 2006-2010 a été considéré comme le point départ réel d'actions gouvernementales en faveur du petit entrepreneuriat (Yared Teshome Geneti, 2018), qui se sont affirmées avec le Growth and Transformation Plan de 2010.

Ces formes d'entrepreneuriat, qui permettent de capter des financements d'institutions internationales, sont aussi un moyen d'encadrer la population urbaine. Le gouvernement s'appuie en effet depuis le milieu des années 2000 sur un dense réseau de partisans qui participent à la stratégie de mobilisation des masses constituant son armée du développement (Lefort, 2016 ; Villanucci \& Fantini, 2016), particulièrement active dans les campagnes (Vaughan \& Tronvoll, $2 \mathrm{OO} 3$; Lefort, 2012 ; Planel \& Bridonneau, 2015 ; Emmenegger, 2016). Ces nouveaux dispositifs en faveur de l'entrepreneuriat associent une sélection des bénéficiaires alors intégrés dans les structures locales de contrôle de la population urbaine (Di Nunzio, 2014 ; Chinigò, 2019). Comme le souligne Di Nunzio (2015), ces politiques ne sont pas le signe d'une adhésion du Parti aux principes du libéralisme, elles ont d'ailleurs des résultats décevants en termes de pérennisation et de soutien des entreprises (Gebrehiwot Ageba et al., 2006). Il s'agit à la fois de faire de l'individu un entrepreneur au service de la communauté (Vaughan, 2011) et de trouver des relais d'embrigadement des populations en faveur du projet de développement du Parti (Chinigò, 2019). Les entrepreneurs ainsi formés sont confrontés à une injonction contradictoire comparable à celle de l'entrepreneur ordonné observé par Honeyman (2016) au Rwanda, devant faire preuve d'une certaine autonomie (self-reliance) tout en se soumettant aux directives et aux contrôles gouvernementaux.

\subsection{Moraliser les entrepreneurs}

L’État développemental constitue également une source de légitimation politique pour le parti au pouvoir (Singh \& Ovadia, 2018) s'accompagnant d'un discours idéologique (Lefort, 2015). Les entrepreneurs, parce qu'ils sont 
supposés privilégier leur intérêt individuel et ne pas se plier de bon gré au cadre idéologique du Parti, sont des acteurs à soumettre pleinement au projet développemental. Chinigò observe ainsi que les formations accompagnant les dispositifs de promotion du petit entrepreneuriat urbain, sont pensées pour inculquer la primauté du travail collectif sur l'individuel (« group first ») (Chinigò, 2019: 86).

L'État, par l'intermédiaire du gouvernement, est conçu non seulement comme un acteur central pour orienter l'économie, mais aussi comme le défenseur de l'indépendance nationale par rapport au secteur privé, en particulier par rapport aux entreprises étrangères (Rodrik, 2004). Les investisseurs étrangers sont donc considérés avec défiance et les espaces de dialogue avec eux sont pensés comme des lieux de moralisation. Le lancement en novembre 2017 d'une plateforme de dialogue sur l'investissement, réunissant les représentants des administrations éthiopiennes sous la houlette de l'Ethiopian Investment Commission (EIC) et ceux d'associations d'investisseurs étrangers, est utilisé pour mettre les entrepreneurs au pas des directives gouvernementales. Un cadre de l'EIC conclut ainsi la première réunion, à la suite d'une demande d'un investisseur de faciliter l'obtention de permis de travail pour les expatriés : «Nous pensons que les investisseurs sont bons dans leur domaine ou pour la valorisation [financière de leur entreprise], mais pas pour eux-mêmes ${ }^{\mathbf{5}}$ » (Fitsum, EIC Commissioner, 29 novembre 2017, traduction de l'auteure). Il considère rendre service aux investisseurs en les encourageant à recruter du personnel local qui leur coûtera beaucoup moins cher que des expatriés.

Dans la droite ligne des principes qui ont présidé à la construction du parti tigréen (Front de libération du peuple du Tigré - TPLF), les entrepreneurs sont considérés comme des « objets » (Lefort, 2016), à qui il faut rappeler leurs devoirs. Ils sont rendus responsables des dysfonctionnements dont ils pâtissent au titre de leur insuffisante volonté à s'intégrer dans les dispositifs publics, de la même manière que les jeunes chômeurs en milieu urbain sont considérés par les pouvoirs publics comme étant responsables de leur pauvreté parce qu'ils ne seraient pas assez travailleurs (Di Nunzio, 2015).

5 "We believe that investors are good for their topic or their valuation, but not for themselves. " 
Qu'il s'agisse d'importants investisseurs étrangers ou de petites entreprises éthiopiennes, la promotion du secteur privé n'existe que parce qu'elle est indispensable au programme de développement de l'État éthiopien et qu'elle sert ses intérêts politiques. Ce cadre ne prévoit aucune place spécifique aux entreprises sociales.

\section{Les entreprises sociales : un projet en friction avec l'État développemental}

À première vue, les entreprises sociales, parce qu'elles mettent au cœur de leur activité une mission qui répond à un impératif de solidarité ou du bien collectif, pourraient sembler trouver une place dans le système promu par le gouvernement éthiopien qui considère l'individu comme étant au service de la communauté et du développement du pays (Vaughan, 2011). Néanmoins, les entreprises sociales en Éthiopie participent à faire du projet entrepreneurial un objectif en soi et non un simple moyen.

\subsection{Des entreprises sociales " hors cadre "}

La lente reconnaissance des entreprises sociales est un constat mondialement partagé, mais il reste qu'elle est plus ou moins avancée selon les pays. Certaines nations ont ainsi adopté des formes juridiques facilitant la reconnaissance de la spécificité de ces structures (Defourny, 2011). Au contraire, en Éthiopie, les entreprises sociales se confrontent à deux cadres juridiques que leurs dirigeants jugent inadaptés à leur activité. Soit les entreprises sociales sont enjointes à se conformer à la Proclamation $n^{\circ}$ 621/2009 (Charities and Society Agency), qui limite la possibilité de générer des revenus (Yntiso Gebre, 2017) et encadre scrupuleusement le budget de ses organisations en restreignant leur possibilité de recevoir des financements de l'étranger'. C'est ce dont témoigne le président de l'association des entreprises sociales éthiopiennes : «Chaque fois que vous avez une idée bénéfique du point de vue social, il est généralement entendu qu'elle doit

6 Cette loi fait suite aux mouvements sociaux de 2005. Elle a été interprétée comme une manière de museler toute critique envers le gouvernement concernant le nonrespect des droits humains (Debebe Hailegebriel, 2010 ; Dupuy et al. 2015). 
être mise en ouvre dans le cadre d'une $\mathrm{ONG}^{\mathbf{7}}$ » (Kibret Abebe interviewé par Birhanu Fikade, 2016, traduction de l'auteure). Soit elles doivent se conformer à la régulation du secteur privé et obtenir une licence de «business ». Dans ce cas, les entreprises sociales sont traitées comme des entrepreneurs « classiques » et leurs dirigeants sont sommés de rentrer dans le rang des politiques publiques en se moulant au modèle du developmental investor tel que dessiné par le gouvernement.

L'expérience de deux entreprises sociales de tissage traditionnel illustre les interprétations concurrentes du projet entrepreneurial. Misant sur des produits haut de gamme, ces entreprises ont développé un atelier permettant d'accueillir des clients internationaux dans un cadre agréable, valorisant le caractère culturel de leur activité, bien loin des parcs industriels standardisés qu'elles sont toutefois encouragées à rejoindre.

Mais le gouvernement nous dit : il faut que vous alliez en zone industrielle. Mais qui va venir nous voir en zone industrielle ? Ici, ce qui est intéressant, c'est le cadre, c'est tout l'environnement. En plus l'existence de village artisanal à côté... [NdA : d'où viennent les salariés]. Pour eux, c'est pas compliqué, ils viennent à pied. Là, il faut qu'ils fassent $30 \mathrm{~km}$ pour aller travailler. Et puis, on n'est pas textile. On n'est pas industrie textile, on n'a pas de machine, mais pour eux, ce secteur quasiment n'existe pas. [...] Bon, ce qu'on fait nous, on peut appeler ça «industrie culturelle», c'est un peu le nouveau terme. (Claude, Français, entreprise de tissage, janvier 2016)

Cette injonction gouvernementale à s'insérer dans un parc industriel ${ }^{\mathbf{8}}$ n'a pas qu'une incidence logistique et géographique, il s'agit aussi de

7 "Whenever you have a socially beneficial idea, the understanding tends to be that it should be entertained via the NGO platform. "

8 La création de parcs industriels en Éthiopie s'inspire des modèles asiatiques et a été décidée par le Growth and Transformation Plan au début des années 2010. Cette politique a largement été portée par une figure politique éthiopienne Arkebe Oqubay, aujourd'hui directeur de l'agence publique des parcs industriels (IPDC). Fin 2019, sur la vingtaine de parcs prévus ou en construction, sept parcs accueillent des entreprises qui ont débuté leurs opérations. Dukem, à 35 km d'Addis-Abeba, est le plus ancien mais est intégralement privé, les autres parcs appartiennent au gouvernement. Sauf Jimma et Dukem, ces parcs sont tous spécialisés dans I'industrie textile ; c'est notamment le cas de Bole Lemmi qui accueille actuellement 23 entreprises à Addis-Abeba et a été le premier parc public opérationnel (en 2015). Les autres parcs sont plus éloignés : Adama est à une heure de route grâce à l'autoroute $(80 \mathrm{~km}$ ), Hawassa (à moins de $300 \mathrm{~km}$ ) a débuté ses activités en 2017 avec 19 entreprises. Kombolcha, dont 3 entreprises avaient commencé leurs opérations 
s'inscrire dans un développement industriel vu comme « moderne », de privilégier la production en quantité permettant le recrutement massif d'une population jeune et en croissance. L'important n'est pas tant de participer au développement que d'y contribuer en suivant les lignes dessinées par le Parti, sous son contrôle.

Dans ce cadre, on comprend mieux l'opposition administrative rencontrée par Aster dans la conduite de son entreprise sociale. Promouvant la consommation de muesli pour lutter contre la malnutrition en Éthiopie, elle a constaté que l'avoine cultivée dans son pays était mélangée avec des graines de blé, nutritionnellement moins bénéfiques. Elle tente donc d'obtenir l'autorisation de faire cultiver de l'avoine dont elle fournirait les semences aux fermiers aux alentours de sa future manufacture :

J'ai dû me battre avec le Ministre, au niveau des wored $a^{9}$ l'année dernière. [...] Ils ne sont pas convaincus. Ils disent : « Pas question! Nous ne [vous soutiendrons pas]. » Il y a un mois, le... un fonctionnaire du service en charge de l'agriculture m'a dit : « Non, non... Comment pouvez-vous oser cultiver cela ? C'est mauvais pour notre pays. C'est mauvais, pas question, pas question ! Je lui ai apporté un petit échantillon. Je lui ai dit : « Vous devez me soutenir. » L'année dernière, ils m’ont dit non. Cette année encore, j'ai apporté un petit échantillon, ils ont dit non. Maintenant, j'ai un autre rendez-vous pour aller au Ministère demain... Aujourd'hui, je les ai appelés, je leur ai dit : «Écoutez, je ne reçois aucun soutien, je veux que les agriculteurs cultivent pour moi, sur une base contractuelle, j'ai obtenu de meilleures semences d'un organisme de recherche, [meilleures] que celles de l'organisme gouvernemental ${ }^{\mathbf{1 0}}$. » (Aster, Éthiopienne, entreprise agroalimentaire, 2019, traduction de l'auteure)

en 2018, est à plus de $350 \mathrm{~km}$ de la capitale. Mekelle, dont 5 entreprises étaient en activité en 2018, est à plus de $700 \mathrm{~km}$. Jimma est enfin un parc spécialisé dans le café et la maroquinerie (à $350 \mathrm{~km}$ d'Addis-Abeba) dont les entreprises ont débuté leur production en 2020

9 Woreda : zone administrative (équivalent d'un discrit), chapeautant plusieurs kebele (quartier regroupant environ 500 foyers).

10 "I have to fight with the minister, at the woreda level last year [...] they're not convinced. They say, 'no way! We won't [support you]'. One month ago, the... an agricultural officer told me 'no, no... How can you dare to grow this? It's damaging to our country. It's damaging, no way, no way!' I brought him a little sample. I said : 'you have to support me'. Last year, they said, no. Again, this year, I brought a little sample, they said no. Now, I have tomorrow, an appointment again to go to the ministry... Today I call them, I said, 'look I don't get any support, I want the farmers to grow for me on a contract basis, I got better seeds from the research organization, [better] than from the government organization'. " 
En voulant recommander cette semence, Aster remet implicitement en question le travail des administrations locales qui contrôlent étroitement l'activité agricole, les engrais dont elles gardent le monopole de la distribution tout comme les semences proposées (Pratten, 1997 ; Planel, 2008). Bref, en revendiquant un positionnement original, les entreprises sociales prennent le risque de rentrer en tension avec le parti, en échappant à son contrôle ou en contredisant ses positions.

\subsection{Promouvoir l'entrepreneuriat de soi au risque d'échapper au contrôle du Parti}

Inscrit dans la tradition anglo-saxonne qui valorise l'entrepreneur en tant qu'individu (Nicholls, 2010), l'entrepreneur social en Éthiopie, tel qu'il se présente dans le discours des enquêtés mais aussi dans les prises de parole des membres de l'association des entreprises sociales éthiopiennes, valorise l'entrepreneuriat de soi (Ehrenberg, 1991). Ce faisant, l'entrepreneuriat est compris comme une fin en soi plutôt qu'un outil au service du projet de développement gouvernemental.

Le récit professionnel de soi, forme de communication plébiscitée des entreprises sociales, est rarement distinct de la narration de la biographie de l'entrepreneur. Un topos classique de l'histoire de la fondation de l'entreprise commence dans le salon, le garage, la cuisine de l'entrepreneur, qui a mobilisé des fonds personnels ou familiaux, bref, qui a engagé au-delà de ses économies la sécurité de sa famille. Par exemple, Kibret Abebe (fondateur d'un service d'ambulances à Addis-Abeba) raconte dans divers entretiens qu'il a vendu sa maison pour pouvoir acheter ses premiers véhicules.

Si la tonalité héroïque est courante dans le récit des/sur les entrepreneurs, qu'ils soient ou non sociaux (Ellis \& Fauré, 1995), ici la valorisation individuelle s'étend aussi aux salariés. C'est particulièrement visible dans les entreprises sociales dont l'objectif porte sur l'accès à l'emploi, via des portraits de leur personnel sur le site Internet ou des publications. Il s'agit de montrer la capacité des entreprises sociales à assurer l'« empowerment » (et la responsabilisation) des populations qui participent à leur projet. Nombre d'entreprises sociales entendent ainsi jouer un rôle éducatif auprès des populations qu'elles emploient, proposant une rationalité concurrente de celle que le gouvernement souhaite voir porter par son armée de développement. 
Rachel, fondatrice d'une des entreprises de tissage citée précédemment détaille ainsi les mesures en termes de formation, au-delà du poste de travail :

\begin{abstract}
Nous avons fait de la formation avant, il y a un très bon organisme de formation pour les femmes ici. Il se concentre sur les femmes, mais nous avons formé nos tisserands et leurs épouses, ou les femmes de leur foyer, leurs épouses et leurs filles, à la gestion des ressources du foyer parce qu'il s'agissait d'essayer de les aider à comprendre que le salaire [versé par l'entreprise] est une ressource pour l'ensemble du ménage. Donc comment pouvez-vous le gérer. Et puis nous avons organisé une formation séparée pour les femmes sur le développement des petites entreprises, et quelques-unes de ces femmes en ont créées ${ }^{11}$. (Rachel, Canadienne, entreprise de tissage, 2018, traduction de l'auteure)
\end{abstract}

Le gouvernement accompagne « ses soldats », citoyens modèles chargés de discipliner leurs voisins et d'encourager des comportements identifiés comme vertueux par les politiques publiques (Villanucci \& Fantini, 2016) en faisant usage de formations similaires (par exemple en valorisant l'épargne...). Cependant, celles dispensées par l'entreprise échappent au système de contrôle du gouvernement. Elles risquent de promouvoir un message concurrent en encourageant l'autonomisation de l'individu par rapport au groupe.

Certes, on peut émettre l'hypothèse que cette démarche renforce l'implication des travailleurs dans des projets communautaires gouvernementaux, tout comme l'attachement à la communauté et la nécessité de participation au développement, principes ancrés depuis plusieurs générations dans l'éducation éthiopienne. Néanmoins, dans les cas les plus extrêmes, cette démarche repose sur l'« empowerment» politique de ces travailleurs, et risque, au contraire, de renforcer les frictions avec un gouvernement autoritaire. Rachel exprime ainsi de manière radicale ce dont ce changement porté par les entreprises sociales en Éthiopie pourrait être synonyme :

Et personnellement, ce que je souhaite, c'est qu'ils deviennent des citoyens vraiment actifs pour leur pays et qu'ils utilisent leur voix pour faire changer

11 "We did training before, there's a really good training organization here for women. Well, it focuses on women, but we trained our weavers and their wives or the women from their households, their wives and daughters whatever, on household resource management because it was trying to help them understand that the salary from [the company] is a household resource. So how can you think about managing that. And then we did a separate training for just the women on small business development ; and a few of the women have started small businesses. " 
les choses. [...] les gens qui sont sûrs de leurs moyens d'existence peuvent en conséquence être ceux qui voteront pour le chef local ou qui deviendront le chef du kebele local ou quelque chose comme ça ${ }^{\mathbf{1 2}}$. (Rachel, Canadienne, entreprise de tissage, 2018, traduction de l'auteure)

Jusqu'à présent, les représentants locaux aux différentes strates administratives sont en effet désignés par le Parti, puis leur candidature est confirmée par une élection. Encourager ses salariés à un engagement citoyen sans référence aux structures du Parti, c'est favoriser la potentielle émergence d'une opposition politique jusqu'à présent largement bâillonnée par le régime.

Le discours partagé de nombreux entrepreneurs sociaux en faveur de « l'empowerment » amène à poser la question de la participation politique, question sensible dans un pays autoritaire. Faute de se plier aux cadres du projet développemental éthiopien, les entreprises sociales doivent donc trouver d'autres ressources institutionnelles pour faire entendre leur voix.

\section{3. À la recherche d'une légitimité institutionnelle : entre extraversion et ancrage}

Les entreprises sociales construisent leur légitimité institutionnelle en Éthiopie en combinant logique d'extraversion qui leur permet d'obtenir une « homologation internationale » (Bayart, 1999), tout en maintenant un ancrage local qui témoigne de la circulation et de l'appropriation des schèmes entrepreneuriaux.

\subsection{L'extraversion pour mobiliser une légitimité internationale}

Lors de la conférence du SEWF (2019), Kibret Abebe, président de l'association des entreprises sociales éthiopiennes, a clairement mis en évidence le rôle de ces entreprises pour transformer le pays et endiguer les situations de pauvreté. S'exprimant ainsi, il peut sembler en ligne avec le discours pro-développement du gouvernement. Pourtant, Bruktawit Tigabu, entrepreneuse sociale éthiopienne à la tête d'une société de production et de

12 "And personally, what I would like, is that they become really active citizens in their country and use their voice to make change. [...] people that are secure in their own livelihood so they could be the people that would vote for local leader or be a local kebele leader or something. " 
réalisation de programmes vidéo éducatifs, et membre du bureau de la même association, affirme, au cours du même événement, que si « ensemble, nous pouvons faire de ce monde un monde meilleur [...]. L'innovation commence en dehors des grandes institutions », qu'il faut savoir être « hors du système qui nous dirige ${ }^{13}$ » (Discours lors du SEWF, Addis-Abeba, octobre 2019, traduction de l'auteure). Navigant entre la recherche d'une institutionnalisation locale (l'association cherche à faire naître un statut juridique pour les entreprises sociales dans le droit éthiopien) et l'affirmation de son indépendance par rapport au gouvernement, les entreprises sociales éthiopiennes usent de l'extraversion pour construire leur légitimité.

Cette dernière repose tout d'abord sur des soutiens financiers, contournant à nouveau l'État éthiopien qui capte habituellement les aides extérieures pour mieux en maîtriser la mise en œuvre (Villanucci \& Fantini, 2016). Nombre d'entreprises sociales ont pu bénéficier de bourses et de financements, notamment via le British Council. Ce dernier les soutient depuis 2013, faisant s'installer dans le pays l'ONG Reach for Change pour y accompagner ses lauréats et dispenser des formations qui visent à transmettre des règles de gestion et de pérennisation d'une entreprise.

Il s'agit ensuite, pour les entreprises sociales, d'aller chercher ailleurs la reconnaissance de leur statut et de leur contribution, déniée ici. Ainsi, des entrepreneurs sociaux se sont fait connaître par la remise de prix financés par des institutions européennes ou américaines. Bethlehem Tilahun Alemu, fondatrice d'une entreprise de confection de chaussures utilisant des semelles de pneumatiques (Solerebels) a été la première entrepreneuse éthiopienne distinguée par la fondation Schwab (Foundation for Social Entrepreneurship) en 2012 comme entrepreneur social de l'année. Bruktawit Tigabu, fondatrice de Whiz Kids, s'est vu décerner la même récompense en 2018. Le British Council soutient également les déplacements des entreprises sociales en les intégrant dans un réseau international qui leur permet de gagner en légitimité de retour en Éthiopie. Comme le souligne Warnier (1993), dans le contexte du Cameroun, le goût pour l'importé renvoie à l'idée de modernité ; ici elle participe à valoriser une forme d'entreprise conçue comme l'avenir du développement économique et social du pays.

13 "Together, we can make this world better [...]. Innovation starts without big institutions " and you need to be " out of the head system. " 
Cette stratégie de légitimation repose enfin sur une forme d'extraversion culturelle (Bayart, 1999). Affirmant leur volonté d'être des agents de changement, les entrepreneurs sociaux s'ancrent dans une tradition américaine qui voit l'entrepreneur comme porteur de changement social (Dees \& Battle Andersen, 2006 ; Bacq \& Janssen, 2011 ; Nicholls, 2010). Cette philosophie est incarnée par Bill Drayton, le fondateur de l'ONG américaine Ashoka, qui soutient les entrepreneurs sociaux qu'elle considère comme des innovateurs de nature à influencer la société. Comme il le souligne lui-même dans une publication, les entrepreneurs sociaux « restent insatisfaits tant que leurs idées n'ont pas changé la société dans son ensemble ${ }^{14}$ » (SSIR, 2004: 11, traduction de l'auteure). Le «bien » comme le changement porté par le mouvement des entreprises sociales, auréolé de son caractère international, est alors conçu comme nécessairement consensuel. L'extraversion permet ainsi « d'aller chercher ailleurs les arguments d'un repositionnement ici », (Fouquet, 2007: 22), confrontant les entrepreneurs à la difficulté d'articuler les échelles globales et locales.

\subsection{L'appropriation comme forme de distinction}

Bruktawit Tigabu n'hésite pas à être critique vis-à-vis des avantages fiscaux que le gouvernement accorde aux investisseurs étrangers, citant les entreprises indiennes et chinoises qui « viennent [faire des affaires] et emmènent tout l'argent en dehors du pays ${ }^{\mathbf{1 5}}$ » (Discours lors du SEWF, AddisAbeba, octobre 2019, traduction de l'auteure). Elle reprend à son compte, d'une part, le discours gouvernemental d'opposition aux entreprises étrangères potentiellement rent-seeker ${ }^{16}$ et, d'autre part, elle revendique une lutte contre l'impérialisme du Nord, suivant un discours développé de longue date au sein de la population éthiopienne.

L'intégration au discours de l'association des entreprises sociales éthiopiennes d'« éléments locaux » constitue alors une stratégie d'appropriation non à destination du gouvernement ou des populations

14 "Are not happy until their ideas have changed the whole society".

15 "Come and take all the money out of the country".

16 Ce terme désigne le comportement d'agents économiques qui visent à rechercher une rente sans que l'activité entreprise n'ait de retombée positive pour le bien-être social ou l'économie globale du pays. 
locales mais comme mode de distinction dans une arène internationale (celle par exemple du Forum mondial des entreprises sociales). Cette stratégie de distinction peut s'observer au sein d'interactions plus ponctuelles rejouant symboliquement les relations de dépendance/domination Nord-Sud. Je rencontre par hasard un représentant de l'association des entreprises sociales éthiopiennes dans un espace de travail partagé (coworking). Cet entrepreneur, réagissant à mes questions sur le soutien du British Council, me fait remarquer que les entreprises sociales ne sont pas une nouveauté en Éthiopie - elles ne sont pas importées. Il cite à l'appui de sa démonstration des formes anciennes de solidarité : d'une part, les Iddir qui sont des associations communautaires prenant en charge l'organisation et les frais des événements sociaux importants comme les funérailles ; d'autre part, les $E q u b$, sorte de tontine à laquelle contribuent à une fréquence déterminée ses membres, un membre bénéficiant généralement de la somme amoncelée par tirage au sort (Gerdes, 1975 ; Pankhurst \& Endreas Eshete, 1958). Peu importe que ces structures aient un lien assez lointain avec les entreprises sociales (Pankhurst, 20o8), il s'agit de ré-ancrer l'entreprise sociale dans le contexte local, par un processus articulant «invention de la tradition » et appropriation d'un concept importé (Bayart, 1999). Dans ce contexte d'énonciation (le représentant se trouve face à une étrangère avec laquelle il s'entretient en anglais), revendiquer une antériorité du concept même d'entreprise sociale, c'est infirmer - si ce n'est renverser - les relations de domination dans le contexte international.

\subsection{Glocalisation et circulation}

Cette invention de la tradition peut prendre différentes formes, notamment ${ }^{\mathbf{1 7}}$ lorsqu'elle est revendiquée par des entrepreneurs étrangers qui souhaitent se distinguer de postures qu'ils identifient comme du néocolonialisme économique. Leur stratégie de glocalisation repose alors sur la valorisation d'un « Ailleurs » qui n'est désirable qu'à l'aune des goûts et aspirations à l'exotisme des consommateurs occidentaux. Souvent sensibles à l'artisanat local, ces entrepreneurs étrangers revisitent les sous-cultures

17 Mais peut-être pas uniquement. Nous l'avons rencontré dans notre échantillon chez les entrepreneurs étrangers, mais différentes entreprises sociales éthiopiennes identifiées mais non encore rencontrées semblent développer le même type d'arguments. 
éthiopiennes, les « modernisent » pour satisfaire une clientèle internationale séduite par la mode « ethnique ». Soul of Africa, financée par Clarks, fabriquant anglais de chaussures, a ainsi lancé une gamme de souliers décorés de motifs « Karo », une ethnie du sud du pays. Les entreprises de Rachel et de Claude déjà citées affichent leur volonté de faire perdurer le savoir-faire éthiopien du tissage. À l'inverse d'autres projets de patrimonialisation en Éthiopie (Josse-Durand, 2015 ; Bridonneau, 2014), ces initiatives échappent totalement au gouvernement, non seulement au sens institutionnel du terme mais aussi au sens cognitif. La relecture de la tradition ne fait pas sens en dehors de son internationalisation. Ces produits ou ces références à des sous-cultures sont généralement négligés par les Éthiopiens euxmêmes car socialement déconsidérés. C'est particulièrement le cas du travail artisanal (Levine, 1974 ; Pankhurst, 2003 ; Mains, 2007) qui a été peu mobilisé politiquement comme «vitrine » du régime ethno-fédéral.

Les poteries, par exemple : on a exporté un conteneur au mois d'août, un conteneur maritime, c'est quand même quelque chose. Peut-être la seule fois dans l'histoire de l'Éthiopie où les poteries sont exportées. Sept mille pièces. Et puis comme il y a environ quatre à cinq semaines de transport, de livraison, on a donné au client deux mois et demi pour payer. On a pris le risque, nous. Mais un jour on envoie notre démarcheur pour exporter les cartons et on lui dit : «Non, vous êtes sur la liste rouge. - Liste rouge ? Comment ça ? - Vous avez exporté un conteneur qui n’a pas été payé, ça veut dire que vous avez été payé en Suisse ou ailleurs. » [...] On est considéré un peu petit peu comme le Gouragué au coin de la rue qui fait du marché noir. Chaque année on exporte... L'année dernière plus d'un million de dollars. Est-ce que le pays a vraiment besoin de devises ou non ? Ça, c'est une décision politique. On a fait passer ce message aussi aux sénateurs américains. (Claude, Français, entreprise de tissage et de poteries)

Les aléas des exportations de Claude illustrent combien cet argument de la valorisation de la culture artisanale éthiopienne convainc peu les élites du pays. En évoquant, d'une part, les Gouragué, ethnie longtemps ostracisée et connue pour son travail artisanal (Pankhurst, 1999), et pour les petites activités commerciales informelles qu'ils réalisent lors de leur migration vers les villes et, d'autre part, le marché noir, Claude fait référence aux marges auxquelles l'administration éthiopienne le renvoie. En retour, il lutte en mobilisant son réseau international (les sénateurs américains, venus visiter son entreprise montée en association avec une Éthiopienne de la diaspora). 
La stratégie de l'extraversion, si elle a permis - ne serait-ce qu'en unifiant sous une même catégorie différentes entreprises appelées « sociales » - une certaine institutionnalisation de l'entreprise sociale, se construit dans un allerretour entre marché international et espaces locaux. Elle ne résout cependant pas les difficultés liées à la conduite d'une telle activité économique dans un contexte autoritaire dans lequel le projet entrepreneurial est en tension avec le plan de développement du gouvernement.

\section{Conclusion}

Pour paraphraser Ellis et Fauré, « les entrepreneurs ne sont ni des vedettes ni des fantômes du développement » mais pourraient en être de « véritables acteurs »(Ellis \& Fauré, 1995: 12). Les entreprises sociales revendiquent explicitement leur capacité à contribuer au développement économique. Cependant, en Éthiopie, elles peinent à articuler leur projet à celui d'un État développemental autoritaire. Les politiques de développement menées par le gouvernement s'accompagnent en effet d'une volonté d'encadrement d'un secteur privé alors que les entreprises sociales encouragent un entrepreneuriat de soi qui constitue une fin plus qu'un outil. Elles prétendent également encourager le changement social, portant les germes d'une libéralisation politique dans les cas les plus radicaux. Pour perdurer dans un environnement peu favorable, elles sont contraintes de suivre la voie étroite de l'extraversion, au risque de renforcer la dépendance du pays aux marchés et aux institutions du Nord.

Quoiqu'elles représentent une petite minorité, les entreprises sociales en Éthiopie illustrent les tensions entre une libéralisation économique contrôlée par l'interventionnisme étatique, d'une part, et un autoritarisme politique qui perdure, d'autre part. Elles laissent voir un autre pan de la glocalisation de l'Éthiopie (Planel \& Bridonneau, 2015), le développement de projets locaux par les entreprises sociales et leur extraversion tendant à court-circuiter le pouvoir fédéral, rapprochant le local et le global.

Enfin, si le capitalisme s'accommode facilement d'un état autoritaire, l'émergence des entreprises sociales pourrait, à certaines conditions, favoriser une certaine libéralisation politique en même temps que le développement 
économique, objectif bien souvent délaissé par les dispositifs d'aide internationale (Brown \& Fisher, 2020 ; Hagmann \& Reyntjens, 2016). Pour se faire, cependant, il faudrait que ces entreprises s'engagent elles-mêmes dans des processus de démocratisation interne qu'elles sont encore une minorité à mettre en œuvre.

\section{L'AUTEURE}

\section{Constance Perrin-Joly}

Constance Perrin-Joly est maîtresse de conférences en sociologie à l'Université Sorbonne Paris Nord, à I'Institut de recherches sur les enjeux sociaux (Iris) et chercheure associée au Centre français des études éthiopiennes (CFEE), à Addis-Abeba. Ses travaux croisent sociologie du travail et analyse des parcours de vie. Ses recherches récentes s'intéressent à l'engagement moral des entrepreneurs en Éthiopie et aux effets de l'industrialisation du pays sur les parcours de vie des travailleur.se.s éthiopien.ne.s.

\section{A récemment publié}

Perrin-Joly, C. (à paraître). Le travail en Éthiopie : rationalisation, dominations, mobilisations. Annales d'Éthiopie, 33.

Collectif. B. (2020). Parler de soi, Méthodes biographiques en sciences sociales. Éditions de l'EHESS.

Perrin-Joly, C. (2020). Faire de la sociologie en/sur l'Éthiopie : institutions, acteur.trices et circulation. Sociologies pratiques, 2(41), Les Presses de Sciences Po.

Perrin-Joly, C. (2020). Entreprendre une enquête, conduire un business en Éthiopie: quand l'incertitude structure le travail de la chercheure en contexte autoritaire. Recherches qualitatives, 39(1), 62-83. https://doi. org/10.7202/1070016ar

Perrin-Joly, C., \& Kushtanina, V. (2018). La composition biographique. Quels effets des choix conceptuels pour saisir les temporalités?. Recherches sociologiques et anthropologiques, 49(2), 115-134. https://doi.org/10.4000/ rsa.2963 


\section{BIBLIOGRAPHIE}

Aalen, L. (2020). The Revolutionary Democracy of Ethiopia: A Wartime Ideology Both Shaping and Shaped by Peacetime Policy Needs. Government and Opposition, 55(4), 653-668. https:// doi.org/10.1017/gov.2018.54

Aalen, L., \& Tronvoll, K. (2009). The End of Democracy? Curtailing Political and Civil Rights in Ethiopia. Review of African Political Economy, 36(120), 193-207. https://doi. org/10.1080/03056240903065067

Altenburg, T. (2010). Industrial Policy in Ethiopia. Discussion Paper. German Development institute.

Aisserge, J., \& Bach, J.-N. (2018). L'Éthiopie d'Abiy Ahmed Ali : une décompression autoritaire. Observatoire de l'Afrique de l'Est, Note Analyse 7. Siences Po/Cedej/DGRIS. https://www. sciencespo.fr/ceri/sites/sciencespo. fr.ceri/files/OAE7-112018.pdf

Alter, S. K. (2002), Case Studies in Social Entrepreneurship. Counterpart International.

Aspen, H. (2020). First Encounters with Industrial Work: Kombolcha Industrial Park. Annales d'Éthiopie, 33.

Bach, J.-N. (2016). L'Éthiopie après Meles Zenawi : I'autoritarisme ethnique à bout de souffle? Politique africaine, 142(2), 5-29. https://doi.org/10.3917/ polaf. 142.0005
Bach, J.-N. (2011). Centre, périphérie, conflit et formation de l'État depuis Ménélik II : les crises de et dans l'État éthiopien $\left(x\left|x^{e}-x x\right|^{e}\right.$ siècles) $)$. Thèse de doctorat, Université de Bordeaux (Lam-IEP).

Bacq, S., \& Janssen, F. (2011). The Multiple Faces of Social Entrepreneurship: A Review of Definitional Issues Based on Geographical and Thematic Criteria. Entrepreneurship \& Regional Development, 23(5-6), 373-403. https:// doi.org/10.1080/08985626.2011.577242

Barraud de Lagerie, P. (2012). Le salaire de la sueur : un éclairage socio-historique sur la lutte anti-sweatshop. Sociologie du travail, 54(1), 45-69. https://doi. org/10.4000/sdt.4303

Barraud de Lagerie, P. (2011). L'affaire Spectrum : la " responsabilité sociale des entreprises " à l'épreuve d'un drame. Revue française de sociologie, 52(2), 245-275. https://doi-org.inshs.bib. cnrs.fr/10.3917/rfs.522.0245

Barrett, P. M., \& Baumann-Pauly, D. (2019). Made in Ethiopia: Challenges in the Garment Industry's New Frontier. NYU Stern Center for Business and Human Rights. https://issuu.com/ nyusterncenterforbusinessandhumanri/ docs/nyu_ethiopia_final_online

Bayart, J.-F. (1999). L'Afrique dans le monde : une histoire d'extraversion. Critique internationale, 5, 97-120. https:// doi.org/10.3406/criti.1999.1505 
Bayart, J.-F. (1989). L'État en Afrique. La politique du ventre. Fayard.

Birhanu Fikade, (2016, July 16). Understanding Social Entrepreneurship. The Reporter. https://www.thereporterethiopia.com/content/understandingsocial-entrepreneurship

Bridonneau, M. (2014). Lalibela, une ville éthiopienne dans la mondialisation. Recompositions d'un espace sacré, patrimonial et touristique. Karthala.

Brown, S., \& Fisher, J. (2020). Aid Donors, Democracy and the Developmental State in Ethiopia. Democratization, 27(2), 185-203. https://doi.org/10.1080/13510 347.2019.1670642

Calvary, R. (2014). Les investissements saoudiens dans la Corne de l'Afrique: I'exemple de Mohamed Al Amoudi, homme d'affaires saoudien en Éthiopie. Confluences Méditerranée, 90(3), 61-74. https://doi.org/10.3917/come.090.0061

Clapham, C. (2018). The Ethiopian Developmental State. Third World Quarterly, 39(6), 1151-1165. https://doi.org/10.10 80/01436597.2017.1328982

Clapham, C. (2006). Ethiopian Development: The Politics of Emulation. Commonwealth and Comparative Politics. 44(1), 137-150. https://doi. org/10.1080/14662040600624536

Clarke, J. (2004). Changing Welfare, Changing States: New Directions in Social Policy. Sage Publications.

Chaponnière, J.-R. (2018). La mutation de l'économie éthiopienne: les limites de la stratégie d'invitation. Afrique contemporaine, 266(2), 55-74. https:// doi.org/10.3917/afco.266.0055
Chinigò, D. (2019). The Peri-Urban Space at Work: Micro and Small Enterprises, Collective Participation, and the Developmental State in Ethiopia. Africa, 89(1), 79-99. https://doi.org/10.1017/ S0001972018000712

Connan, D. (2014). La destinée manifeste de l'entrepreneuriat kényan : charité et constitution morale d'une élite économique africaine. Politix, 108(4), 123-141. https://doi.org/10.3917/pox.108.0121

Dart, R. (2004). The Legitimacy of Social Enterprise. Nonprofit Management \& Leadership. 14(4), 411-424. https:// doi.org/10.1002/nml.43

Debebe Hailegebriel (2010). Restrictions on Foreign Funding of Civil Society. International Journal of Not-for-Profit Law, 12(3), 18-27.

Defourny, J., Nyssens, M. (2010). Conceptions of Social Enterprise and Social Entrepreneurship in Europe and the United States: Convergences and Divergences. Journal of Social Entrepreneurship, 1(1), 32-53. https://doi. org/10.1080/19420670903442053

Defourny, J. (2011). L'émergence et le développement du concept d'entreprise sociale. In Amadio, N. (Ed.). Les dynamiques de l'entrepreneuriat social (21-44). Néothèque.

Dees, G., \& Battle Anderson, B. (2006). Framing a Theory of Social Entrepreneurship: Building on Two Schools of Practice and Thought. ARNOVA. https://centers.fuqua.duke.edu/case/ wp-content/uploads/sites/7/2015/02/ BookChapter_Dees_FramingTheoryofSE_2006.pdf 
Dereje Feyissa (2012). Aid Negotiation: The Un Easy 'Partnership' Between EPRDF and the Donors. Journal of Eastern African Studies, 5(4), 788-817. https:// doi.org/10.1080/17531055.2011.642541

De Waal, A. (2013). The Theory and Practice of Meles Zenawi. African Affairs, 112(446), 148-155. https://doi. org/10.1093/afraf/ads081

Di Nunzio, M. (2015). What is the Alternative? Youth, Entrepreneurship and the Developmental State in Urban Ethiopia. Development and Change, 46(5), 1179-1200. https://doi.org/10.1111/ dech.12187

Di Nunzio, M. (2014). Thugs, Spies and Vigilantes: Community Policing and Street Politics in Inner City Addis Ababa. Africa, 84(3), 444-465. https:// doi.org/10.1017/S0001972014000357

Dupuy, K., Ron, J., \& Prakash, A. (2015). Who survived? Ethiopia's Regulatory Crackdown on Foreign-Funded NGOs. Review of International Political Economy, 22(2), 419-456. https://doi. org/10.1080/09692290.2014.903854

Ehrenberg, A. (1991). Le Culte de la performance. Hachette.

Ellis, S., \& Fauré, Y.-A. (1995). Entreprises et entrepreneurs africains. Karthala.

Emmenegger, R. (2016). Decentralization and the Local Developmental State: Peasant Mobilization in Oromiya, Ethiopia. Africa, 86(2), 263-287. https:// doi.org/10.1017/S0001972016000048
Fantini, E., \& Puddu, L. (2016). Ethiopia and International Aid: Development Between High Modernism and Exceptional Measures. In Hagmann, T., \& Reyntjens, F. (Eds.). Aid and Authoritarianism in Africa: Development without Democracy. Zed Books.

Fouquet, T. (2007). De la prostitution clandestine aux désirs de l'Ailleurs: une " ethnographie de l'extraversion " à Dakar. Politique africaine, 107(3), 102-123. https://doi.org/10.3917/ polaf.107.0102

Gebrehiwot Ageba, Wolday Amha, Getnet Alemu, Alemayehu Seyoum, Alemu Mekonnen, Eyob Tesfaye, Ayele Gezahegn, \& Diwan Ishak (2006). Micro and Small Enterprises (MSE) Development in Ethiopia: Strategy, Regulatory Changes and Remaining Constraints. Ethiopian Economics Association, 10(2), 103-118. https://doi.org/10.22004/ ag.econ. 249856

Gerdes, V. (1975). Precursors of Modern Social Security in Indigenous African Institutions, The Journal of Modern African Studies, 13(2), 209-228. https:// www.jstor.org/stable/160190

Hagmann, T., \& Reyntjens, F. (2016). Aid and Authoritarianism in Africa: Development without Democracy. Zed Books.

Hamel, J. (2000). À propos de l'échantillon. De l'utilité de quelques mises au point. Recherches qualitatives, 21, 3-20. http://mtis2.ds.iscte.pt/10-11/ recursos $\% 20$ didcticos/amostras $\% 20$ na\%20pesquisa\%20qualitativa.pdf 
Honeyman, C. A. (2016). The Orderly Entrepreneur: Youth, Education, and Governance in Rwanda. Standford University Press.

Huybrechts, B., Nicholls, A. \& Mouchamps, H. (2012). Entrepreneuriat social : définitions, ressorts et défis. In Bayle, E. (Ed.). Management des entreprises de l'économie sociale et solidaire : identités plurielles et spécificités (89-106). De Boeck Supérieur.

Johnson, C. (1982). MIT and the Japanese Miracle: The Growth of Industrial Policy, 1925-1975. Stanford University Press.

Josse-Durand, C. (2015). Le musée Konso au cœur de l'arène : quand les courtiers en développement (re)dessinent les contours du champ politique éthiopien. EchoGéo, 31. https://doi.org/10.4000/ echogeo.14144

Kerlin, J. A. (2006). Social Enterprise in the United States and Europe: Understanding and Learning from the Differences. Voluntas: International Journal of Voluntary and Nonprofit Organizations, 17, 246-262. https://doi.org/10.1007/ s11266-006-9016-2

Lefort, R. (2016). La crise de I'EPRDF : "Devenir inclusif!". Politique africaine, 142(2), 121-144. https://doi.org/10.3917/ polaf.142.0121

Lefort, R. (2015). The Ethiopian Economy. The Developmental State vs the Free Market. In Prunier, G., \& Ficquet, E. (Eds.). Understanding Contemporary Ethiopia: Monarchy, Revolution and the Legacy of Meles Zenawi (357-394). Hurst.
Lefort, R. (2012). Free Market Economy, 'Developmental State' and Party-State Hegemony in Ethiopia: The Case of 'Model Farmers'. The Journal of Modern African Studies, 50(4), 681-706. https:// doi.org/10.1017/S0022278X12000389

Levine, D. (1974). Greater Ethiopia: The Evolution of a Multiethnic Society. The University of Chicago Press.

Le Velly, R. (2014). Entrepreneuriat social. In Chauvin, P. M., Grossetti, M., \& Zalio, P. P., (Eds.). Dictionnaire sociologique de I'entrepreneuriat (191-203). Les Presses de Sciences Po. https://halshs.archivesouvertes.fr/halshs-01096407/document

Mains D. (2007). Neoliberal Times: Progress, Boredom, and Shame among Young Men in Urban Ethiopia. American Ethnologist, 34(4), 659-673. https://doi. org/10.1525/ae.2007.34.4.659

Mains D. (2012). Blackouts and Progress: Privatization, Infrastructure, and a Developmentalist State in Jimma, Ethiopia. Cultural Anthropology, 27(1), 3-27. https://doi.org/10.1111/j.15481360.2012.01124.x

Morelle M. \& Planel S. (2018). Appréhender des " situations autoritaires". Lectures croisées à partir du Cameroun et de l'Éthiopie. L'espace politique, 35(2). https://doi.org/10.4000/espacepolitique.4902

Nallet, C. (2019). Les orientations libérales d'Abiy Ahmed. Sauvetage économique ou tournant idéologique ?. Éditoriaux de I'IFRI, 47. https://www.ifri.org/sites/ default/files/atoms/files/nallet_ethiopie_ abiy_ahmed_2019_002_1.pdf 
Nicholls, A. (2010). The Legitimacy of Social Entrepreneurship: Reflexive Isomorphism in a Pre-Paradigmatic Field. Entrepreneurship Theory and Practice, 34(4), 611-633. https://doi.org/10.1111/ j.1540-6520.2010.00397.x

Oqubay Arkebe (2015). Made in Africa : Industrial Policy in Ethiopia. Oxford University Press.

Pankhurst, A. (2008). The Emergence, Evolution and Transformations of Iddir Funeral Associations in Urban Ethiopia. Journal of Ethiopian Studies, 41(1-2), 143-185.

Pankhurst, A (2003). Introduction: Dimensions and Conceptions of Marginalisation. In Freeman, D., \& Pankhurst, A. (Eds.). Peripheral People: The Excluded Minorities of Ethiopia (1-26). Red Sea Press.

Pankhurst, A. (1999). "Caste" in Africa: The Evidence from South-Western Ethiopia Reconsidered. Africa, 69(4), 485-509. https://doi.org/10.2307/1160872

Pankhurst, R., \& Endreas Eshete (1958). Self-help in Ethiopia. Ethiopia Observer, 2(11), 354-336.

Planel, S. (2008). La chute d'un Éden éthiopien. Le Wolaita, une campagne en recomposition. IRD Éditions.

Planel, S., \& Labzaé, M. (2018). La République fédérale démocratique en marche : crise politique, état de grâce et avancée néolibérale en Éthiopie. Politique africaine, 150(2), 161-178. https://doi.org/10.3917/polaf.150.0161
Planel, S., \& Bridonneau, M. (2015). Glocal Ethiopia. Échelles et repositionnements des pouvoirs. EchoGéo, 31.

Pratten, D. T. (1997). Local Institutional Development and Relief in Ethiopia: A Kire-based Seed Distribution Programme in North Wollo. Disasters, 21(2), 138-154. https://doi.org/10.1111/14677717.00050

Ramo, J. C. (2004). The Beijing Consensus. The Foreign Policy Centre. https:// fpc.org.uk/publications/the-beijingconsensus/

Rodrik, D. (2004). Industrial Policy for the 21th Century. CEPR Discussion Paper, 4767. http://cemi.ehess.fr/docannexe/ file/2738/rodrik_2004.pdf

Singh, J. N. \& Ovadia, J. S. (2018). The Theory and Practice of Building Developmental States in the Global South. Third World Quarterly, 39(6), 1033-1055. https://doi.org/10.1080/01436597.201 8.1455143

Stevenson, L., \& St-Onge, A. (2005). Support for Growth-oriented Women Entrepreneurs in Ethiopia, Kenya and Tanzania: An Overview Report. ILO. https://www.ilo.org/public/libdoc/ ilo/2005/105B09_30_engl.pdf

SSIR (Stanford Social Innovation Review) (2004). " 15 minutes with Bill Drayton", Stanford Social Innovation Review, 2. https://ssir.org/articles/entry/15_ minutes_with_bill_drayton 
Teasdale, S. (2011). What's in a Name? Making Sens of Social Enterprises Discourses. Publics Policies and Administration, 27(2), 99-119. https:// doi.org/10.1177/0952076711401466

Vaughan, S. (2011). Revolutionary Democratic State-building: Party, State and People in the EPRDF's Ethiopia. Journal of Eastern African Studies, 5(4), 619-640. https://doi.org/10.1080/17531 055.2011 .642520

Vaughan, S., \& Mesfin Gebremichael (2011). Rethinking Business and Politics in Ethiopia: The Role of EFFORT, the Endowment Fund for the Rehabilitation of Tigray. Africa Power and Politics Research Report, 2.

Vaughan, S. \& Tronvoll, K. (2003). The Culture of Power in Contemporary Ethiopian Political Life. Sida Studies, 10. https://gsdrc.org/document-library/ the-culture-of-power-in-contemporaryethiopian-political-life/

Villanucci, A., \& Fantini, E. (2016). Santé publique, participation communautaire et mobilisation politique en Éthiopie : la Women's Development Army. Politique africaine, 142(2), 77-99. https://doi. org/10.3917/polaf.142.0077

Warnier, J-P. (1993). L'esprit d'entreprise au Cameroun. Karthala.
Yared Teshome Geneti (2018). Challenges and Opportunities of Micro and Small Enterprises Strategy in Ethiopia Urban Development: The Case of Ambo Town, Oromia, Ethiopia. SDMIMD Journal of Management, 9(1), 55-64. https://doi. org/10.18311/sdmimd/2018/19994

Yntiso Gebre (2017). Reality Checks: The State of Civil Society Organizations in Ethiopia. African Sociological Review, 21(1), 20-42. https://www.jstor.org/ stable/90013859

Young, J. (1997). Peasant Revolution in Ethiopia. The Tigray People's Liberation Front, 1975-1991. Cambridge University Press.

Young, D., \& Salamon, L. (2002). Commercialization, social ventures, and for-profit competition. In Salamon, L. (Ed.). The State of Non-Profit America (425-448). Brookings Institution Press.

Zenawi Meles (2012). States and Markets: Neoliberal Limitations and the Case for Developmental State. In Noman, A., Botchwey, K., Stein, H., \& Stiglitz, J. E. (Eds.). Good Growth and Governance in Africa: Rethinking Development Strategies. Oxford University Press.

Zewde \& Associates (2002). Women Entrepreneurs in Ethiopia. Report for ILO Office Addis-Ababa. ILO. 


\title{
- L'entrepreneuriat en Afrique
}

\author{
Sous la direction de Quentin Chapus, Jean-Philippe Berrou \\ et Yvette Onibon Doubogan
}

- Introduction : le retour du héros ?

L'entrepreneur, itinéraire d'un concept chez les « développeurs » en Afrique Quentin Chapus - Jean-Philippe Berrou - Yvette Onibon Doubogan

\section{DOSSIER}

- Un entrepreneuriat local à l'ombre de la politique industrielle en Éthiopie Les résidents étrangers depuis 1940

David Ambrosetti - Dominique Harre

- Vous avez dit « entrepreneuriat »?

Quand conceptions et intérêts divergent sur le terrain, dans le département du Mono, Bénin

Maïté Kervyn de Lettenhove - Andreia Lemaître

- La formation à l'entrepreneuriat de soi dans l'université marocaine Salwa Hanif - Rhita Sabri

- Les entreprises sociales à la recherche de leur légitimité

Frictions autour du projet de développement éthiopien et tentatives d'extraversion Constance Perrin-Joly

- Des Nana Benz aux cheffes d'entreprise contemporaines à Lomé, au Togo L'entrepreneuriat dit « moderne » en question Charlotte Vampo

- PME agroalimentaires et entrepreneuriat inclusif au Burkina Faso Entre opportunisme, recherche du profit et lutte contre la pauvreté Florent Song-Naba - Philippe Régnier

\section{VARIA}

- Le Génie militaire au Cameroun

Une force duale pour l'aménagement du territoire et le développement Axel Augé - Christel Dior Tamegui

\section{LE DÉVELOPPEMENT DANS LES MÉDIAS}

- La médiatisation de l'entreprise et de l'entrepreneuriat en Afrique Laëtitia Larcher

\section{ANALYSES BIBLIOGRAPHIQUES}

